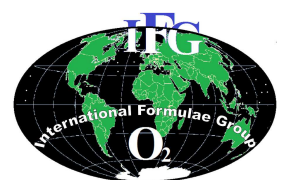

Available online at http://www.ifg-dg.org

Int. J. Biol. Chem. Sci. 10(2): 793-807, April 2016

International Joumal

of Biological and

Chemical Sciences

ISSN 1997-342X (Online), ISSN 1991-8631 (Print)

Original Paper http://ajol.info/index.php/ijbcs http://indexmedicus.afro.who.int

\title{
Engagements contractuels et performances des organisations de producteurs de maïs à l'Ouest Cameroun
}

\author{
Guillaume Hensel FONGANG FOUEPE ${ }^{1 *}$, Denis Pompidou FOLEFACK ${ }^{2}$ \\ et Eugenie JUEGOUO NGON PISSIE ${ }^{1}$
}

${ }^{1}$ Faculté d'Agronomie et des Sciences Agricoles, Université de Dschang, B.P 222 Dschang Cameroun.

${ }^{2}$ Ministère de la Recherche Scientifique et de l'Innovation, B.P. 1457 Yaoundé, Cameroun.

*Auteur correspondant ; E-mail : guillaumefongang@yahoo.fr

\section{RESUME}

Cet article met en exergue le rôle des engagements contractuels dans l'amélioration des performances des organisations des producteurs (OP) à l'Ouest Cameroun. Il est basé sur des enquêtes par questionnaires auprès des délégués de 33 OP de maïs bénéficiaires de l'encadrement du Programme d'Amélioration de la Compétitivité Agricole (PACA). L'analyse statistique à partir des moyennes et des fréquences permet d'aboutir aux résultats qui indiquent l'existence de contrats d'achats d'intrants et de commercialisation du maïs. Ces contrats permettent aux OP d'obtenir des intrants de meilleure qualité à des prix inférieurs de 4 à $17 \%$ par rapport aux prix d'achat hors contrats. Aussi ces contrats permettent aux OP d'accéder à un marché plus lucratif et de réaliser des gains supplémentaires avec des prix au kg de maïs supérieur de $12 \%$ à ceux pratiqués hors contrats. Toutefois, la durabilité de tels contrats et de leurs impacts n'est pas garantie dans la mesure où les OP de maïs concernées ont bénéficié d'un accompagnement important du PACA dans leurs démarches. Ainsi, la présence du PACA a un effet de garantie car rassurant les acheteurs de maïs et fournisseurs d'intrants. L'impact de tels contrats en l'absence de structures d'appui aux OP mérite d'être étudié pour réellement en apprécier la viabilité et les effets. Aussi, il est nécessaire d'étudier les conditions de minimisation des risques dans de telles démarches contractuelles.

(c) 2016 International Formulae Group. All rights reserved.

Mots clés : Contrats, agriculteurs, commercialisation, partenariats, chaine de valeur, Ouest-Cameroun.

\section{Contractual agreement and maize producers' organisations' performances in West Cameroon}

\begin{abstract}
This article highlights the role of contractual agreement on the improvement of producers' organisations (POs) performances in the West region of Cameroon. The study is based on survey with the delegates of 33 maize POs receiving support from the Agricultural Competitiveness Project (PACA). Statistical analysis through average and frequency enables us to obtain results showing the existence of input purchases' contracts and maize marketing agreement. Thanks to these contracts, OPs are able to access high quality inputs at better price from 4 to $17 \%$ lower than prices without contract. Also, these contracts enable POs to access to
\end{abstract}


more lucrative market and earn an extra $12 \%$ on the selling price of each kilogram of maize as compared to the price without contract. However, the sustainability of these contracts and their impacts are not guarantee as maize POs concerned have been receiving substantial support from PACA in their strategies. Therefore, the presence of PACA as a guarantee effect by comforting inputs suppliers and maize buyers. The impact of these contracts without supporting structure like PACA need to be studied in order to really appreciate their viability and effects. Likewise, it is necessary to study risks minimization conditions in such a contractual approach.

(C) 2016 International Formulae Group. All rights reserved.

Keywords: Contracts, farmers, marketing, partnerships, value chain, West Cameroon.

\section{INTRODUCTION}

La croissance démographique et l'urbanisation rapide constituent des évolutions majeures récentes des savanes d'Afrique Centrale. Ces évolutions se manifestent dans un contexte international caractérisé par le désengagement des États, la libéralisation croissante des économies nationales ainsi que des mutations imprévisibles dont les enjeux majeurs sont la lutte contre la pauvreté et l'insécurité alimentaire (Touzard et Temple, 2012). L'ouverture du monde rural sur la ville et l'accroissement de la population ont toutefois favorisé des stratégies paysannes d'adaptation centrées sur la diversification des productions vivrières et un engouement pour les produits porteurs. Le maïs et bien d'autres cultures ont bénéficié, grâce aux appuis institutionnels substantiels, de cet essor du vivrier marchand, passant de culture de consommation familiale à une culture de marché. Au cours de ces dernières années, la culture du maïs tend à devenir une culture commerciale comme le café ou le cacao en procurant des revenus non négligeables aux producteurs. L'intégration croissante de ces grains dans les habitudes alimentaires a engendré un accroissement remarquable des surfaces et volumes de production (Ndjouenkeu et al., 2010 ; Fofiri et al., 2010).

Le maïs, céréale la plus cultivée au Cameroun, est consommé régulièrement par quelque 12 millions de personnes, soit les deux tiers de la population. C'est la principale source de revenus de plus de trois millions de petits exploitants agricoles camerounais. Après le blé et le riz, le maïs est la céréale la plus répandue dans le monde, en jouant un rôle prépondérant dans la sécurité alimentaire des populations. Il contribue également pour plus de 150 milliards de FCFA au Produit Intérieur Brut du Cameroun (Ntsama et Kamgnia, 2008).

Malheureusement, l'expansion de la filière maïs au Cameroun fait face à plusieurs difficultés à savoir: des techniques de production rudimentaires, une faible productivité du travail et une faible organisation des acteurs. Ces difficultés entraînent une évolution de la production qui est largement inférieure à l'accroissement de la population. Par ailleurs, la faible productivité des producteurs de maïs, adjointe à la demande croissante en provenance des agro-industries et des pays voisins, contribue à accentuer le déficit d'offre nationale du maïs. Ce gap de production est d'autant plus préoccupant que, les rendements de maïs obtenus en milieu paysan restent faibles (1,5 à 2,6 tonnes.ha $\left.^{-1}\right)$ et sont en général inférieurs de $50 \%$ à $80 \%$ aux rendements optimums facilement accessibles avec les technologies rendues disponibles par la recherche. Malgré les politiques agricoles mises en place pour stimuler la production agricole, il y'a toujours une insatisfaction de la couverture de la demande en produits agricoles, en particulier de la demande en maïs. En 2009, l'Association Camerounaise pour la Défense des Intérêts Collectifs (ACDIC) estimait la demande nationale annuelle de maïs à 
1500000 tonnes pour une production de 1380000 tonnes soit un déficit de 120000 tonnes (ACDIC, 2011).

$\mathrm{Au}$ regard de l'enjeu que revêtent les cultures comme le maïs, le gouvernement camerounais a entrepris, en collaboration avec ses partenaires au développement dont la Banque Mondiale, d'initier le Projet d'Amélioration de la Compétitivité Agricole (PACA) au Cameroun en 2010. Le projet est cofinancé par l'Etat Camerounais et la Banque Mondiale pour un montant total de 82 millions de dollars dont $73 \%$ (soit 60 millions de FCFA) par la Banque Mondiale. Son objectif est de développer et d'accroître la compétitivité des organisations de producteurs agropastoraux opérant dans les filières agropastorales considérées comme stratégiques du point de vue de leur contribution à la sécurité alimentaire, de leurs avantages comparatifs sur les marchés régionaux et de leur contribution à la croissance (MINADER/MINEPIA, 2009).

L'augmentation de l'offre du maïs sur les marchés urbains de la région de l'OuestCameroun soulève quelques enjeux de fonds relatifs à : l'organisation des acteurs et au fonctionnement des activités pour la compétitivité de la filière, l'accroissement des revenus des acteurs en terme de valorisation de la production et de pérennisation de l'offre permanente en maïs, la sécurité alimentaire d'une population régionale croissante et de plus en plus exigeante sur la qualité des produits.

Dans un contexte de libéralisation et de désengagement de l'Etat, les organisations de producteurs ont un rôle prépondérant à jouer dans la filière maïs. Dans le but d'accroître les performances des OP de maïs, le PACA promeut des partenariats économiques en organisant des fora qui regroupent des organisations paysannes agricoles, les producteurs de semences, les fournisseurs d'intrants agricoles, les éleveurs, les commerçants des produits agricoles et d'élevage. L'objectif à travers ces fora est de créer un cadre d'échange, de favoriser des accords entre les agriculteurs et les autres acteurs de la filière et d'aider au renforcement des capacités. Ces accords pouvant par la suite conduire à des accords interprofessionnels qui sont, selon Broutin et al. (2008), des éléments performants permettant d'organiser une filière, les marchés et de faciliter les relations entre les acteurs et même avec l'Etat. Depuis la mise en place de ces initiatives en 2010, la question fondamentale que l'on se pose est celle de savoir si les partenariats économiques ainsi initiés ont contribué à l'amélioration des performances des organisations de producteurs de maïs bénéficiaires et ont permis d'accroître significativement la production $\mathrm{du}$ maïs dans les zones d'intervention. Bien que la littérature sur la filière maïs soit abondante, très peu d'auteurs se sont intéressés sur les questions d'engagements contractuels entre acteurs.

L'insatisfaction à l'égard des théories traditionnelles du fonctionnement du marché a conduit les économistes à explorer la portée de la notion de contrat. La notion de contrat fournit les bases d'une théorie dans laquelle les dispositifs de coordination résultent des réactions décentralisées et rationnelles des agents. Elle se substitue à la notion de marché walrasien, le système économique étant perçu comme un immense réseau de contrats.

Le contrat est un accord par lequel des parties se créent des obligations mutuelles afin de se coordonner, c'est-à-dire rendre leurs actions compatibles et partager le surplus d'utilité ainsi créé. Ces contrats ne sont pas nécessairement «écrits » ou des documents juridiques. Il s'agit d'accords interindividuels qu'ils soient écrits ou non, explicites ou implicites (Salanie, 1994 ; Brousseau, 1997). Cette extension, somme toute relativement récente, autorise d'expérimenter son application au contexte rural africain, par exemple à la production cotonnière où domine encore la tradition de l'oral. 
Le contrat occupe aujourd'hui une place centrale dans l'analyse de la coordination car c'est un concept essentiel dans la conception libérale des mécanismes d'harmonisation des intérêts individuels dans une société décentralisée. Paradoxalement, les économistes s'en sont historiquement peu servis pour étudier les propriétés d'une économie décentralisée, préférant recourir à une approche, celle du marché walrassien dans laquelle, la coordination n'est pas interindividuelle puisque l'offre et la demande se rencontrent à un niveau agrégé. Les limites de cette dernière approche ont conduit à renouer avec la notion de contrat au cœur de la pensée libérale sur les ressorts du fonctionnement d'une société décentralisée (Brousseau, 1993 ; Brousseau, 1997).

Trois principales raisons expliquent le succès de l'approche «contractuelle» des dispositifs de coordination. Premièrement, le contrat apparaît comme le dispositif de coordination le plus simple à étudier. En effet, il est fréquemment envisagé comme bilatéral et présente une structure moins complexe que celles qui assurent la coordination entre un grand nombre d'acteurs. Deuxièmement, cette notion est en adéquation avec l'individualisme méthodologique; la genèse et la forme des dispositifs de coordination étant expliquées à partir des comportements individuels. Troisièmement, et résultant en partie du point précédent, l'approche peut s'appuyer sur les outils traditionnels de la théorie néoclassique, d'où une grande puissance analytique comme en témoigne le succès des applications des théories des contrats à l'analyse du marché du travail ou des méthodes de réglementation (Perrot, 1992 ; Laffont et Tirole, 1993).

En Afrique francophone, cette question de coordination contractuelle est très peu étudiée par les économistes. Dans une perspective sociologique et d'économie institutionnelle, Fongang (2004) mobilise le concept de dispositif territorial, une espèce d'engagement contractuel pluri-acteurs à l'échelle d'un territoire. Quelques rares auteurs, à l'instar de Hugon (2005, 2007), se sont également penchés sur cette question. Il souligne que, l'approche microéconomique insiste sur la rationalité «située» des agents qui préfèreront souvent une situation satisfaisante à une situation optimale. Dans un monde complexe et imprévisible, il est difficile de prévoir toutes les éventualités et les adaptations possibles. C'est pourquoi, les contrats incomplets du point de vue de la théorie économique mais basés sur des relations de confiance entre acteurs sont le plus souvent préférés. Ainsi, dans le cas du contexte africain, les contrats implicites sont l'équivalent des accords non contraignants de la théorie des jeux et permettent d'éviter les effets de cavalier libre ou favorisent des comportements efficients.

L'objectif de ce travail a été d'analyser les effets des engagements contractuels sur les performances des organisations de producteurs de maïs dans la région de l'OuestCameroun.

\section{MATERIEL ET METHODES \\ Zone d'étude}

L'étude a été réalisée au Cameroun et principalement dans la région de l'Ouest. C'est une zone de forte production agricole, le maïs étant l'une des principales spéculations cultivées. De plus, des engagements contractuels entre les organisations de producteurs et les autres acteurs de la filière maïs y existent déjà, d'où le choix de cette région comme zone d'étude.

\section{Collecte des données}

Des données de sources primaires ont été collectées à l'aide d'un questionnaire administré à 33 délégués d'organisations de producteurs de maïs encadrées par le PACA dans les départements du Noun et des Bamboutos. Ces deux départements sont les grands bassins de production du maïs à l'Ouest-Cameroun (Fongang, 2008), d'où leur 
choix. Dans ces départements, un échantillon de 33 organisations de producteurs sur les 81 de la région dont 22 sur 49 dans les Bamboutos et 11 sur 31 dans le Noun a été constitué. Les critères de sélections des OP étaient les suivants: être une organisation paysanne ayant bénéficié du cofinancement du PACA, avoir pour activité principale la production de maïs et avoir signé des contrats agricoles. En plus des enquêtes, des observations directes ont été faites sur le terrain. Les données collectées portaient principalement sur: les caractéristiques des OP, leurs activités, les enjeux des contrats entre acteurs, la typologie des contrats, l'accès aux intrants, les coûts des intrants, les quantités de maïs produit, le mode de commercialisation du maïs produit, les conditions de mise en place des contrats, etc.

\section{Analyse statistique}

Les données ont été analysées à l'aide du logiciel SPPS, permettant d'obtenir des statistiques descriptives, principalement les fréquences et les moyennes.

\section{RESULTATS}

Quelques caractéristiques des organisations de producteurs de maïs enquêtées

Les organisations des producteurs (OP) sous engagement contractuel sont tous des Groupements d'Initiatives Communes légalisées pour la plupart entre 2000 et 2005 (49\%), et entre 2006 et 2010 (33\%). Si la production et la commercialisation du maïs constituent les principales activités de ces OP, leurs membres pratiquent également l'élevage du porc et de la volaille (33\%).

Les surfaces cultivées sont importantes (57\% des OP exploitent plus de 20 hectares) et sont soit des champs communautaires, soit des parcelles individuelles dans lesquelles les membres mutualisent leurs ressources pour produire. Ces superficies importantes entraînent une forte demande en intrants pour la production ainsi qu'une importante offre en maïs. Ce qui peut justifier et faciliter la recherche des partenaires avec qui signer des contrats, et ainsi établir la pertinence des contrats. Toutefois, ces OP s'exposeraient à d'importantes pertes au cas où les partenaires avec qui ils signent des contrats venaient à ne pas remplir leurs engagements.

Les membres des OP enquêtés sont des personnes prédisposées à faire partie des groupes de producteurs et ambitieuses quant à une perspective en grande partie marchande de l'agriculture. Ils ont majoritairement (63\%) un âge compris entre 36 et 45 ans et entre 26 et 35 ans (22\%), avec une moyenne d'âge de 40 ans. Par ailleurs on y retrouve sensiblement la même proportion d'hommes $(49,6 \%)$ que de femmes $(50,4 \%)$.

La majorité (73\%) des OP enquêtés ont un effectif compris entre 11 et 20 membres, $24 \%$ ont plus de 20 membres et $3 \%$ moins de 11 membres. Ces membres constituent la main d'œuvre dans les exploitations agricoles. Ceci limite les surfaces potentiellement susceptibles d'être mises en valeur par une OP ou entraîne des coûts supplémentaires du fait du recours dans certains cas à la main d'œuvre agricole rémunérée. Aussi, dans les OP, le niveau de scolarisation des membres semble relativement bas; la majorité $(58 \%)$ ayant le primaire pour niveau d'étude le plus élevé. Le Tableau 1 présente les caractéristiques des enquêtés suivant leur niveau d'étude.

\section{L'organisation des fora d'acteurs d'une filière : objectifs et finalités}

A l'Ouest-Cameroun, face aux difficultés rencontrées par les producteurs pour accéder aux intrants et commercialiser les produits agricoles, le PACA a initié des fora. Les fora étant des rencontres organisées en des lieux précis, et regroupant les organisations paysannes agricoles, les producteurs de semences, les fournisseurs d'intrants agricoles, les éleveurs et les commerçants dans le but de promouvoir des accords de partenariat concernant la 
commercialisation des produits agricoles des organisations paysannes agricoles et l'approvisionnement de ces dernières en intrants.

Les fora visent à promouvoir entre les acteurs mobilisés des relations durables et mutuellement gagnantes par le biais de contrats. Parmi les objectifs recherchés, ces fora doivent permettre aux producteurs et aux OP de :

- avoir accès aux semences améliorées, aux engrais de qualité et de trouver des débouchés sûrs pour la commercialisation de leur production et à des prix rémunérateurs ;

- nouer des partenariats économiques matérialisés par des contrats agricoles. Des contrats qui permettront par la suite aux producteurs individuels et à leurs organisations de créer des relations mutuellement bénéfiques et durables avec les autres acteurs de la filière.

\section{Typologie des contrats entre les OP et les acteurs de la filière maïs}

Dans le cadre des engagements contractuels entre acteurs de la filière maïs, l'étude a permis de distinguer cinq types de contrats qui pouvaient être signés à savoir : les contrats d'achat d'engrais, d'achat de fientes de poules, d'achat de semences, de commercialisation et de production.

Les contrats d'achat (d'engrais, fientes et semences) sont établis entre une ou plusieurs OP avec le ou les fournisseurs les plus compétitifs (moins cher) rencontrés lors des fora, parfois après passation de marché. Dans ces contrats, les fournisseurs s'engagent à fournir les intrants faisant l'objet du contrat (engrais, semences ou fientes) en quantité, à prix, en qualité, en lieu et date convenus. Dans certains cas, il peut arriver que les fournisseurs assurent la livraison du produit au siège de l'OP. Quant à l'OP, elle s'engage à s'acquitter du paiement du produit selon les modalités de paiement prévues par le contrat, soit par virement bancaire, soit au comptant.
Par ailleurs, dans le cas des contrats d'achat de semences, les semences fournies doivent être certifiées par les services étatiques de contrôle et de certification. Par ailleurs, il est à noter que le maïs sert de moyen de paiement pour certains contrats, notamment entre les OP et les fournisseurs de fientes de poules.

Les contrats de commercialisation sont établis entre les OP et des acheteurs (éleveurs, producteurs de bières locales, commerçants grossistes, etc.). Ici, l'OP s'engage à mettre à la disposition de l'acheteur un produit (le maïs) avec des caractéristiques précises (calibre et couleurs des graines, taux d'humidité, quantités, etc.) suivant des modalités de livraison définies dans le contrat. En contrepartie, l'acheteur s'engage à effectuer le paiement à l'OP suivant les modalités prévues dans le contrat (par virement bancaire ou au comptant, en paiement unique ou en plusieurs tranches, etc.). Ce contrat est un document écrit portant les engagements des contractants.

Les contrats de production s'apparentent en réalité aux contrats de commercialisation, à la différence que, contrairement à ces derniers, ils sont établis avant le début de la campagne agricole. C'est ainsi que, en plus des accords régulièrement retrouvés dans des contrats de commercialisation, les contrats de production peuvent prévoir que l'acheteur assure des services comme la fourniture des intrants, l'octroi de crédit ou un appui technique à l'OP. Le Tableau 2 présente le nombre d'OP ayant signé chaque type de contrat dans le cadre des partenariats économiques promus par le PACA à l'Ouest-Cameroun entre 2010 et 2012 .

Toutes les OP enquêtées ont donc déjà eu à établir des contrats relatifs à l'achat de fientes de poules, d'engrais et de semences. De l'avis de ces OP, ces contrats sont très avantageux. Lorsqu'elles passent ces contrats, elles ont la possibilité d'acheter ces intrants à crédit. En plus, à cause du volume important 
des commandes des OP et de la concurrence entre les fournisseurs, les prix proposés par les fournisseurs sont inférieur par rapport au prix sur le marché. A titre d'exemple, la différence est de 1000 FCFA par sac d'urée et de 1200 FCFA par sac d'engrais NPK (14-24-14).

D'autre part, seuls $18 \%$ des OP dans le Noun et $63 \%$ de celles dans les Bamboutos, soit $48 \%$ de toutes les OP enquêtées ont déjà établi des contrats de commercialisation. Très généralement, les OP espèrent avoir de meilleurs prix d'achats de maïs que ceux qui leur sont proposés avant la commercialisation, et préfèrent ainsi vendre au moment venu. Aucune des OP rencontrées n'a eu à établir des contrats de production; les risques de production étant très élevés du fait que, les producteurs n'ont pas une grande maitrise de la production.

Performance des organisations des producteurs sous engagements contractuels

Le but premier des organisations des producteurs ayant opté pour des engagements contractuels pour la production du maïs est l'amélioration de leur performance financière. Ce qui passe en partie par une réduction considérable des coûts de production, une amélioration de la gestion post récolte des produits, une amélioration des prix de vente et une maximisation du profit des exploitations agricoles (individuelle et collective).

Des engrais de meilleure qualité obtenus à travers les contrats

La comparaison du niveau d'appréciation des engrais utilisés par les OP entre la situation de référence (sans contrat) en 2010 et les engrais achetés lors du forum en 2012 permet de dresser le Tableau 3.

L'analyse du Tableau 3 révèle que, $58 \%$ des enquêtés jugent la qualité des engrais obtenues sous contrat très bonne alors que, seuls $6 \%$ de ces derniers estiment qu'elle l'était déjà avant l'avènement des contrats. Avec les contrats, aucun enquêté ne juge les engrais qui lui ont été fournis comme étant de mauvaise qualité. Ces contrats ont donc permis une amélioration nette et significative de la qualité des engrais fournis aux OP. En effet, selon les enquêtés, avant les contrats, les engrais faisaient l'objet de contrefaçon. A ce moment-là, les OP s'approvisionnaient chez des détaillants dans les marchés. Or, ces derniers ont l'habitude de substituer une partie de l'engrais dans les sacs par une substance la ressemblant physiquement. Alors que, actuellement, les engrais proviennent directement des magasins du fournisseur sans intervention des intermédiaires. Par conséquent, grâce aux contrats, les producteurs ont désormais accès à des engrais de meilleure qualité.

Un accès aux intrants à des prix abordables selon les $\mathrm{OP}$

Selon les enquêtés, les contrats agricoles ont aussi contribué à l'acquisition des intrants agricoles à des prix meilleurs. Les OP n'achètent plus leurs intrants de façon individuelle au prix du marché mais de façon groupée et au prix des fora. Le Tableau 4 compare le prix de quelques intrants avec contrat et sans contrat.

Il ressort du Tableau 4 que, le prix moyen du sac de $50 \mathrm{~kg}$ d'engrais NPK acheté suite à un contrat est de 18829 FCFA contre 19524 FCFA sur le marché. Ainsi, une OP sous contrat gagne en moyenne 695 FCFA (4\% du prix en achat individuel) par sac d'engrais NPK acheté. De même, pour l'urée, il obtient un gain de 815 FCFA (4\% du prix sans contrat) par sac de $50 \mathrm{~kg}$. Pour les fientes, le gain est de 275 FCFA par sac; soit $11 \%$ du prix individuel. Finalement, pour les semences de maïs, le gain est de 110 FCFA (17\%) par kg de semences achetés.

Le prix n'est pas une variable permettant de mesurer à lui seul l'efficacité d'un achat, d'autres charges comme les frais de transport et les coûts de transaction devant aussi être pris en considération. Toutefois 
l'établissement de contrats d'achats par les OP a permis de réduire les prix d'achat des intrants. Cette réduction à l'unité doit être appliquée aux quantités acquises collectivement et à celles revenant aux individus pour apprécier la valeur globale de la réduction ainsi obtenue. Les économies ainsi faites peuvent alors être réinvesties dans d'autres activités de l'OP ou permettre de couvrir d'autres charges de production $y$ compris celles relatives au transport, aux négociations, etc. Comme autre conséquence de cette amélioration dans l'accès aux intrants, une augmentation des surfaces cultivées par les OP est observée. Ainsi, on est passé de $62 \%$ d'OP cultivant moins de 20 hectares en 2010 à seulement $42 \%$ en 2012.

Amélioration du prix de vente du maïs avec les engagements contractuels

La maximisation du profit à travers la vente des produits agricoles est l'objectif de toute activité de production. Les OP de maïs trouvent dans la signature de contrats une occasion non seulement de vendre leurs produits à des prix meilleurs, mais aussi de réduire en partie les coûts supplémentaires liés aux ventes individuelles. Le Tableau 5 présente et compare le prix moyen de vente du kilogramme de maïs sur le marché en cas de vente individuelle et à celui obtenu lorsqu'un contrat de vente est établi.

Une différence moyenne de 22 FCFA est obtenue entre le prix moyen de vente du $\mathrm{kg}$ de maïs sur le marché et celui de vente sous contrat, soit une augmentation de $14 \%$ du prix avec les contrats. En effet, avec l'appui du PACA, les OP maïs ont amélioré leur pouvoir de négociation et ont de plus en plus accès à des marchés plus lucratif grâce aux accords établis lors des fora. Par ailleurs, les acheteurs en ressortent aussi gagnant dans la mesure où, cela leur permet d'épargner un travail de collecte à travers plusieurs achats individuels et de gagner ainsi du temps qui peut être réinvesti dans d'autres activités.

La durabilité des contrats établis avec les organisations de producteurs

Les contrats permettent aux OP d'acquérir des intrants de bonne qualité et en quantité. Cependant, tout ceci n'auraient sans doute pas été possible si ces OP n'avaient reçu des financements du PACA.

Les contrats sont établis et réussissent en grande partie grâce à un acteur de l'ombre, le PACA qui joue le rôle de garantie permettant aux fournisseurs de s'assurer que les paiements seront effectifs. Les achats d'intrants à crédit par les OP ne seraient certainement pas possible sans la présence du PACA. Ces observations interrogent sur la viabilité et la continuité de ces contrats en l'absence d'un acteur facilitateur et agissant comme garant à l'exemple du PACA. Ceci interpelle quant à la nécessité de développer avec les institutions de financement des mécanismes de garantie au profit des OP dans de telles opérations. On peut en effet se demander si les OP peuvent d'elles-mêmes disposer d'éléments de garantie pouvant permettre aux fournisseurs de s'engager dans de telles transactions avec des paiements à crédit.

De même, la durabilité de ces contrats ne peut être garantie que si les différences de prix (prix des intrants sous contrat par rapport aux prix sur le marché) sont significatives aux yeux des OP. Une analyse ultérieure devrait permettre de mieux mesurer la contribution des contrats indépendamment de l'aspect subvention par le PACA. Car, il s'agit en réalité de contrats suscités par le PACA, signés avec son appui et dont les paiements sont effectués grâce à son financement octroyé sous forme de don aux OP. 
Tableau 1 : Niveau de scolarisation moyen des membres des OP enquêtés.

\begin{tabular}{|c|c|c|c|c|c|c|}
\hline \multirow[b]{2}{*}{ Départements } & \multicolumn{5}{|c|}{ Niveau scolarisation moyen des membres de l'OP } & \multirow[b]{2}{*}{ Tota } \\
\hline & Pas été à l'école & Niveau primaire & Premier cycle secondaire & $\begin{array}{l}\text { Second cycle } \\
\text { secondaire }\end{array}$ & Supérieur & \\
\hline Bamboutos & 0 & 13 & 2 & 7 & 0 & 22 \\
\hline Noun & 0 & 6 & 2 & 3 & 0 & 11 \\
\hline Total & 0 & 19 & 4 & 10 & 0 & 33 \\
\hline$\%$ & 0 & 58 & 12 & 30 & 0 & 100 \\
\hline
\end{tabular}

Tableau 2 : Nombre d'OP par type de contrats agricoles à l'Ouest Cameroun entre 2010 et 2012.

\begin{tabular}{|c|c|c|c|c|c|c|}
\hline \multirow[t]{2}{*}{ Types de contrats } & \multicolumn{6}{|c|}{ Nombre d'OP (\% OP) } \\
\hline & \multicolumn{2}{|c|}{ Bamboutos } & \multicolumn{2}{|c|}{ Noun } & \multicolumn{2}{|c|}{ Total } \\
\hline Contrats d'achat de semences & 22 & 100 & 11 & 100 & 33 & 100 \\
\hline Contrats de commercialisation & 14 & 63 & 2 & 18 & 16 & 48 \\
\hline Contrats d'achat de fientes de poules & 22 & 100 & 11 & 100 & 33 & 100 \\
\hline Contrats de production & 0 & 0 & 0 & 0 & 0 & 0 \\
\hline
\end{tabular}


Tableau 3 : Comparaison du niveau d'appréciation des engrais chimiques achetés par les OP en 2010 (situation de référence) et ceux achetés sous contrat en 2012.

\begin{tabular}{lcc}
\hline Appréciation des OP & Situation de référence (2010) & Situation sous contrat (2012) \\
\cline { 2 - 3 } & \multicolumn{2}{c}{ Pourcentage (\%) } \\
\hline Mauvaise & 9 & 0 \\
Bonne & 85 & 42 \\
Très bonne & 6 & 58 \\
Total & 100 & 100 \\
\hline
\end{tabular}

Tableau 4 : Moyenne et écart-type du prix des engrais NPK en achat groupé et en achat individuel.

\begin{tabular}{lccccr}
\hline Type d'intrants & Moyenne & Ecart type & Minimum & Maximum & Effectif \\
\cline { 2 - 6 } & \multicolumn{5}{c}{ Prix (FCFA) } \\
\hline Sac de NPK (achat groupé) & 18829 & 1296 & 16550 & 23000 & 33 \\
Sac de NPK (achat individuel) & 19524 & 1363 & 17750 & 25000 & 33 \\
Sac d'Urée (achat groupé) & 18960 & 1505 & 17460 & 20465 & 33 \\
Sac d'Urée (achat individuel) & 19775 & 1552 & 18165 & 20885 & 33 \\
Sac de fiente (achat groupé) & 2280 & 197 & 2085 & 2480 & 33 \\
Sac de fiente (achat individuel) & 2555 & 195 & 2365 & 2750 & 33 \\
Semences (achat groupé) & 530 & 46 & 485 & 575 & 33 \\
Semences (achat individuel) & 640 & 71 & 570 & 710 & 33 \\
\hline Un sac $=50 \mathrm{~kg}$ & & & & &
\end{tabular}

Tableau 5 : Comparaison des prix moyens de vente du maïs sur le marché par rapport aux prix moyens du maïs en cas de contrat de vente.

\begin{tabular}{lrrrr}
\hline \multicolumn{1}{c}{ Produits } & \multicolumn{4}{c}{ Prix (FCFA/Kg) } \\
\cline { 2 - 5 } & Moyenne & Ecart type & Minimum & Maximum \\
\hline Maïs (vente direct sur le marché) & 157 & 28 & 100 & 249 \\
Maïs (vente par contrat) & 179 & 20 & 117 & 215 \\
\hline
\end{tabular}

\section{DISCUSSION}

Les contrats agricoles, une source d'efficience et d'efficacité pour les OP

A l'Ouest-Cameroun, le développement des cultures vivrières $\mathrm{y}$ compris le maïs fait suite à une reconversion des agriculteurs vers le vivrier marchand et le maraîchage après la déprise caféière. Pour plus d'efficacité dans leurs actions, les producteurs vont s'organiser en OP avec pour activité principale la production et la commercialisation des produits vivriers (Uwizeyimana, 2009). Afin de promouvoir les contrats agricoles, Cai et al. (2008) affirme 
que les organisations de producteurs peuvent jouer un rôle important en renforçant leur acceptabilité politique et sociale. Ce qui peut expliquer le recours du PACA à ces dernières pour promouvoir les contrats agricoles en milieu rural. De plus, Agronomes et Vétérinaires Sans Frontières (AVSF) (2014) précisent que la présence d'une organisation de producteurs (OP) est souvent une condition favorable et pérenne aux dynamiques d'agriculture contractuelle, car elle permet de rééquilibrer les relations de négociations, peut aider à parvenir à des accords plus précis, plus équitables et donc qui seront mieux respectés.

Il est observé que, les OP de maïs sous engagement contractuel dans la région de l'Ouest acquièrent des intrants en quantité suffisante et de meilleure qualité à des prix compétitifs (efficience) et à crédit. Les contrats d'approvisionnement en intrants (semences, engrais, fientes) sont d'ailleurs ceux que nous avons le plus rencontrés. Un résultat qui va dans le sens de ceux observés dans la filière maïs en Equateur où, il a été constaté que, les producteurs cherchaient en premier, dans les programmes mis en œuvre par l'Etat et ses partenaires et dans leurs relations contractuelles avec les entreprises, à avoir accès aux semences de maïs et autres intrants, et au service de crédit car ils n'avaient en général pas de trésorerie propre pour l'achat d'intrants pour la production, ni d'accès au crédit bancaire (AVSF, 2014).

De plus, elles vendent leur récolte à des prix meilleurs (efficacité). Ce qui, au sens de Jacquet (2011), fait de ces OP des structures performantes car, selon cet auteur, une entreprise (entendue ici par OP) est performante lorsqu'elle atteint ses objectifs fixés (efficacité) à moindre coût (efficience). Prowse (2013) a affirmé d'ailleurs que, l'adoption des contrats agricoles par les OP s'inscrit dans une recherche d'efficacité et d'efficience. En outre, nos observations corroborent ceux de Prowse (2013) et Vavra (2009) qui soulignent que, les agriculteurs contractualisés ont des revenus sensiblement plus élevés que les autres. Une étude réalisée en Inde a également montré que, la production sous contrat était généralement beaucoup plus rentable que la production hors contrat (Government of India, 2007).

Une faible tendance au recours aux contrats de production

La signature d'un contrat agricole implique que, l'exploitant agricole s'engage à produire selon certaines modalités un produit que l'acheteur s'engage à acheter (Minot, 2007; Vavra, 2009; Prowse, 2013). Une garantie que n'offrent pas les producteurs camerounais et qui pourrait expliquer l'absence des contrats de production et la faible représentativité des contrats de commercialisation. En effet, ces producteurs ne disposent en général d'aucunes maitrises sur les facteurs externes (pédoclimatiques) et encore moins des ressources et technologies nécessaires pour la production, la manutention et même le stockage des produits. Il est par conséquent difficile pour eux de garantir aux potentiels acheteurs la disponibilité du produit en quantité et qualité adéquate aux moments convenus. Il serait donc important que des efforts soient faits pour un changement de mentalités et une appropriation de la culture des contrats de commercialisation par les acteurs concernés. Le tout premier est d'accroître le niveau de maîtrise des itinéraires de production, des techniques de gestion post récoltes et des stratégies d'adaptation aux aléas climatiques par les OP de maïs.

Par ailleurs, le niveau d'étude relativement faible des responsables des OP de maïs ne les prédisposent pas à suffisamment d'analyse stratégique, d'astuces de négociation et peut de ce fait constituer un handicap pour la durabilité des contrats; une incompréhension, un vice de fond ou de forme et une non-exécution des clauses de contrat pouvant facilement survenir et causer des 
conflits entre les différents partenaires. Or, selon Hamilton (2012), il est important que les producteurs soient à même de comprendre le contenu des contrats agricoles et soient à même de les négocier pour leur réussite. Il est donc nécessaire que soient renforcées leurs capacités de négociations afin d'éviter des situations où des contrats seraient conclus au détriment des agriculteurs comme en Inde où de pareils cas ont été recensés (Government of India, 2007). De plus, les contrats agricoles étant une innovation dans le paysage agricole camerounais, ce niveau d'éducation relativement bas constitue un handicap pour leur adoption; le taux adoption d'une innovation étant affecté par le niveau d'instruction de sa cible (Rogers, 1995).

D'autre part, le non recours au contrat de production s'explique par le comportement opportuniste des OP et acheteurs potentiels. En effet, l'OP peut vendre son produit hors contrat (si un autre acheteur lui fait une proposition plus lucrative) ou encore l'acheteur peut résilier le sien afin d'acheter un produit à un prix plus bas sur le marché. Aucune partie ne veut donc prendre le risque de se faire léser. De plus les OP ne sont pas encore prêtes à prendre le risque de vendre leur produit à un prix bas par rapport à celui sur le marché ; le prix de vente étant défini à l'avance grâce à des spéculations basées sur l'évolution des prix des années antérieures et des aléas climatiques (avant la production). De même, il est rare de trouver des acheteurs prêts à prendre le risque d'acheter un produit à un prix supérieur au prix sur le marché. En somme, la difficulté pour les deux parties est celle de s'entendre sur un prix incitatif pour les agriculteurs. L'acheteur s'arrangeant en général à ne pas prévoir payer plus que le prix estimé par lui pour la période de livraison tandis que, dans le même temps, le producteur espère toujours que les prix sur le marché seront supérieurs le moment venu à celui proposé par le potentiel acheteur. Des observations qui traduisent le comportement d'agents économiques devant prendre des décisions pour le futur tout en étant dans une situation d'incertitude et de manque de précision sur les données prévisionnelles d'aide à la décision. Pourtant, Vavra (2009) suggère l'intervention de tierces parties, qui définiraient et négocieraient les contrats en entreprenant des actions qui réduiraient les coûts de transaction ; contribueraient à combler les lacunes en matière d'information dans la négociation des contrats ; faciliteraient l'exécution des contrats et la réduction des fraudes ; et limiteraient les possibilités de « hold-up » et de recherche de rente. Ces arbitres devant aussi intervenir pour que la bonne volonté de part et d'autre ne cède pas à la méfiance et aux griefs (Prowse, 2013). C'est donc ce rôle qui est en partie dévouée au PACA. Le PACA, constituant à titre d'exemple, pour les OP, une caution morale face aux fournisseurs dans le but de garantir l'accès à des services agricoles. L'intervention du PACA comme tierce partie devrait aussi avoir pour conséquence de renforcer les OP, de les aider à créer un climat de confiance et faire respecter l'accord, faciliter les processus avec des subventions, extensions de taxes et impôts ou des facilités d'exportation, fixer un cadre légal pour les contrats, veiller au respect des engagements et faciliter la résolution des conflits (AVSF, 2014).

\section{Conclusion}

Dans la région, plusieurs types de contrats ont été établis entre les OP et certaines entreprises opérant dans la filière maïs. Une typologie des contrats montre des contrats d'achats d'engrais (NPK et Urée), d'achats de fientes, d'achats de semences et des contrats de commercialisation. Bien que possible, les contrats de production n'ont pas encore été établis par les OP à cause de 
comportements opportunistes des acteurs de la filière et la rationalité limitée de ces derniers. Par ailleurs, les contrats ont permis aux OP d'avoir accès à des intrants de meilleure qualité, en quantité suffisante et à des prix réduits. Ces derniers ont permis aux producteurs de réaliser des économies sur les achats pouvant aller de $4 \%$ pour l'achat des engrais à $17 \%$ pour l'achat des semences.

En outre, avec les contrats, les producteurs ont amélioré leur pouvoir de négociation en effectuant des ventes groupées leur permettant d'avoir accès à un marché plus lucratif grâce aux accords établis lors des fora. C'est ainsi qu'elles peuvent vendre leur récolte à un prix supérieur de 22 FCFA par $\mathrm{kg}$ à celui sur le marché, soit une augmentation de près de $12 \%$.

Toutefois, il serait judicieux pour les OP de ne plus se limiter au prix (d'achat et vente) pour juger l'efficacité d'une transaction mais, de prendre aussi en compte les charges supplémentaires (frais de transport, coût de transaction, risque) qu'elles supportent pour acquérir les intrants ou pour vendre leurs récoltes. De même, les études ultérieures sur la contribution des contrats sur les performances des OP devraient considérer ces éléments. Mais dans l'immédiat, les OP devraient négocier les conditions avantageuses (livraison gratuite, suivi technique après l'achat, etc.) lors de la signature des contrats afin de s'assurer une bonne profitabilité de pareilles opérations.

L'expérience du PACA interpelle tout en montrant la nécessité d'actions d'appui en facilitation à une meilleure insertion des petits producteurs et leurs organisations dans une économie de marché compétitive. Une étude sur la durabilité des contrats entre les acteurs de la filière maïs dans cette région méritent d'être conduite afin d'évaluer l'impact réel de ces contrats sur le développement de la filière maïs indépendamment de l'appui du PACA.
De même, il est nécessaire que soit étudié les conditions de sécurité et de minimisation des risques dans les démarches contractuelles encouragés par le PACA.

\section{CONFLIT D'INTERET}

Les auteurs déclarent qu'ils n'ont aucun conflit d'intérêt

\section{CONTRIBUTION DES AUTEURS}

GHFF a contribué à la collecte et analyse des données, et à la rédaction de l'article. DPF a contribué à l'enrichissement théorique et conceptuel, à la rédaction de l'article. EJNP a contribué à l'réalisation des enquêtes et participation à l'analyse des données et à la rédaction de l'article.

\section{REFERENCES}

Agronomes et Vétérinaires Sans Frontières. 2014. A Quelles Conditions l'Agriculture Contractuelle Peut-Elle Favoriser les Agricultures Paysannes du Sud ? Edition Coordination SUD Solidarité Urgence Développement: Paris ; 56.

Association Citoyenne de Défense des Intérêts Collectifs. 2011. Données Statistiques Des Importations Des Produits et Aliments de Base de 2004 à 2011. ACDIC: Yaoundé, Cameroun.

Brousseau E. 1997. Théories des contrats, coordination interentreprises et frontières de la firme. In Les Frontières de la Firme, Garrouste $\mathrm{P}$ (ed). Economica : Paris.

Brousseau E. 1993. L'Economie des Contrats: Technologies de l'Information et Coordination Interentreprises. PUF : Paris.

Broutin C, Duteurtre G, Liagre L, Teyssier J. 2008. Diversité, Enjeux et Conditions de Succès des Organisations 
Interprofessionnelles. Grain de Sel : Paris, France.

Cai J, Lung S, Setboonsarng, Leung P. 2008. Rice Contract Farming in Cambodia: Empowering Farmers to Move Beyond the Contract Toward Independence. ADBI discussion paper, $\mathrm{N}^{\circ}$ 109. Institut de la Banque Asiatique de Développement : Tokyo.

Eaton C, Shepherd AW. 2002. L'agriculture contractuelle. Des partenariats pour la croissance. Bulletin des services agricoles 145 de la FAO. FAO : Rome.

Fofiri Nzossie EJ, Temple L, Ndame JP, Dury S, Ndjouenkeu R, Simeu Kamdem M. 2010. L'émergence du maïs dans la consommation alimentaire des ménages urbains au Nord-Cameroun. Revue Economie Rurale, Paris, France ; 318-319.

Fongang Fouepe GH. 2010. Évolution et défis du mouvement paysan au Cameroun. Grain de sel $\mathrm{n}^{\circ} 49$, janvier - mars 2010. Paris, France.

Fongang Fouepe GH. 2008. Les Mutations du Secteur Agricole Bamiléké (Cameroun) étudiées à travers ses acteurs : Une analyse des localités de Fokoué et de Galim. Thèse de Doctorat : Institut des Sciences et Industries du Vivant et de l'Environnement (Agro Paris Tech), Paris, France, p 415

Fongang Fouepe GH. 2004. Dynamiques des Dispositifs Territoriaux: le cas du dispositif agri-environnemental en Corse. Mémoire Diplôme d'Etudes Approfondies, Option : «Territoires, Sociétés et Environnement », Institut National Agronomique Paris-Grignon et Institut National de la Recherche Agronomique. Paris, France, p 140
Government of India. 2007. Report of the working group on agricultural marketing infrastructure and policy required for internal and external trade for the XI Five year plan 200712. Agriculture division, planning commission, Government of India: New Delhi.

Hamilton ND. 2008. Agricultural Contracting: a U.S. perspective and issues for India to consider. In Contract farming in India: a resource book, Gulat, A, Joshi PK, Landes M (eds.).

Hugon P. 2007. Méso Analyse de Filières et Politiques Publiques, Illustration par les Filières Cotonnières en Afrique, Concept et Méthodes en Economie des Filières. CIRAD : Montpellier, France.

Hugon P. 2005. Les filières cotonnières africaines au regard des enjeux nationaux et internationaux. Notes et Etudes Economiques, 23: 87-112.

Laffont JJ, Tirole J. 1993. A Theory of Incentives in Procurement and Regulation. MIT Press: Cambridge.

MINADER/MINEPIA. 2009. Manuel de suivi évaluation du Projet d'Amélioration de la compétitivité Agricole (PACA). Ministère de l'Agriculture et du développement Rural : Yaoundé.

Minot N. 2007. Contract Farming in Developing Countries: Patterns, Impact, and Policy Implications. Etude de cas 6-3 du programme Food Policy for Developing Countries: the Role of Government in the Global Food System. Cornel University.

Ndjouenkeu R, Fofiri NEJ, Kouebou C, Njomaha C, Grembombo, Miam O K. 2010. Le maïs et le niébé dans la sécurité alimentaire urbaine des savanes d'Afrique centrale. ISDA 
2010, Montpellier du 28 juin $-1^{\text {er }}$ juillet 2010. 17 p.

Ntsama E, Kamgnia DSM. 2008. Les déterminants de l'adoption des variétés améliorées de maïs : adoption et impact de la «CMS 8704 ». Université de Yaoundé II : Soa, Cameroun.

Perrot A. 1992. Les Nouvelles Théories du Marché du Travail. Repères : Paris.

Prowse M. 2013. L'Agriculture Contractuelle dans les Pays en Développement une Revue de Littérature, A Savoir, 12. Agence Française de Développement : Paris. p 110

Rogers E.M. 1995. The Diffusion of Innovations (4th edn.). The Free Press: New York.
Salanie B. 1994. Théorie des Contrats. Economica : Paris.

Touzard JM, Temple L. 2012. Sécurisation alimentaire et innovations dans l'agriculture et l'agroalimentaire : vers un nouvel agenda de recherche? Une revue de la littérature, Synthèse. Cahiers Agricultures, 21(5): 293301.

Uwizeyimana L. 2009. Après le café, le maraîchage? Mutations des pratiques agricoles dans les Hautes Terres de l'Ouest Cameroun. Les Cahiers d'Outre-Mer, 247 : 331-344.

Vavra P. 2009. L'Agriculture Contractuelle : Rôle, Usage et Raison d'Etre. Éditions OCDE; p43. 